\title{
A SHAPE OPTIMIZATION PROBLEM FOR STEKLOV EIGENVALUES IN OSCILLATING DOMAINS
}

\author{
JULIÁN FERNÁNDEZ BONDER AND JUAN F. SPEDALETTI
}

\begin{abstract}
In this paper we study the asymptotic behavior of some optimal design problems related to nonlinear Steklov eigenvalues, under irregular (but diffeomorphic) perturbations of the domain.
\end{abstract}

\section{INTRODUCTION}

Let $\Omega \subset \mathbb{R}^{n}$ be a bounded domain with regular boundary, let $\alpha \in(0,1)$ and $\Gamma \subset \partial \Omega$ be a measurable set (a window) such that $|\Gamma|_{n-1}=\alpha|\partial \Omega|_{n-1}$, where $|\cdot|_{d}$ refers to the $d$-dimensional Hausdorff measure. The optimal Sobolev trace constant is defined as

$$
\lambda(\Gamma):=\inf _{v \in W_{\Gamma}^{1, p}(\Omega)} \frac{\int_{\Omega}|\nabla v|^{p}+|v|^{p} d x}{\int_{\partial \Omega}|v|^{p} d S},
$$

where $W_{\Gamma}^{1, p}(\Omega)$ is the set of functions $v \in W^{1, p}(\Omega)$ such that $\left.v\right|_{\Gamma}=0$.

Observe that a minimizer for $\lambda(\Gamma)$ always exists thanks to the compactness of the embedding $W^{1, p}(\Omega) \subset \subset L^{p}(\partial \Omega)$ and that any minimizer $u$ of $\lambda(\Gamma)$ is a weak solution of the following Steklov-type eigenvalue problem

$$
\begin{cases}-\Delta_{p} u+|u|^{p-2} u=0 & \text { in } \Omega \\ u=0 & \text { on } \Gamma \\ |\nabla u|^{p-2} \frac{\partial u}{\partial \mathbf{n}}=\lambda(\Gamma)|u|^{p-2} u & \text { on } \partial \Omega \backslash \Gamma,\end{cases}
$$

where $\mathbf{n}$ is the unit outer normal of $\partial \Omega$. Moreover, $\lambda(\Gamma)$ is the first (principal) eigenvalue of this problem. See [9].

In [5], the authors study the following problem: minimize $\lambda(\Gamma)$ among all admissible windows, i.e.

$$
\lambda(\alpha)=\inf _{\Gamma \in \Sigma_{\alpha}} \lambda(\Gamma),
$$

where $\Sigma_{\alpha}=\left\{\Gamma \subset \partial \Omega\right.$ : measurable and $\left.|\Gamma|_{n-1}=\alpha|\partial \Omega|_{n-1}\right\}$.

In the above mentioned work the authors show the existence of an optimal window $\Gamma_{0}$, i.e. some $\Gamma_{0} \in \Sigma_{\alpha}$ such that $\lambda(\alpha)=\lambda\left(\Gamma_{0}\right)$. Moreover it is shown that if $u_{0}$ is an eigenfunction associated to $\lambda\left(\Gamma_{0}\right)$ then $\left\{u_{0}=0\right\} \cap \partial \Omega=\Gamma_{0}$.

2010 Mathematics Subject Classification. 35P30, 35J92, 49R05.

Key words and phrases. Shape optimization, Steklov eigenvalues, Gamma convergence, Oscillating domains. 
We refer the interested reader to [5] and references therein for a motivation and history of this problem. See also [6] for some related optimization problem.

In this work we study the behavior of this optimal windows when the domain $\Omega$ is perturbed periodically by a sequence of domains $\Omega_{\varepsilon}$ and try to determine whether they approximate $\Gamma_{0}$ in some reasonable sense.

The interest in problems with oscillating boundary appears in the influence of micro-structures of surfaces (porous medium, composites, micro-materials) over the large scale behavior. The mathematical analysis of problems with oscillating boundary was presented in [11].

Let us denote by $\lambda_{\varepsilon}(\alpha)$ the constant (1.1) in the domain $\Omega_{\varepsilon}$. We find that the behavior of the constants $\lambda_{\varepsilon}(\alpha)$ and of their corresponding optimal windows $\Gamma_{\varepsilon}$ depend strongly on the amplitude of the oscillations. We distinguish three cases: i.- Subcritical case: in this case the oscillations are very big and the trace constant converges to zero. ii.- Supercritical case: in this case the oscillations are very small and there are convergence to the unperturbed problem. iii.- Critical case: in this case the amplitude compensates with the oscillations and this is reflected in the appearance of a weight term.

The results presented here are new even in the linear eigenvalue problem that corresponds to $p=2$.

1.1. $\varepsilon$-Oscilations. In [5], the authors studied the asymptotic behavior of $\lambda_{\varepsilon}(\alpha)$ where the domains $\Omega_{\varepsilon}$ are regular perturbations of the original domain $\Omega$. To be precise, the authors apply the so-called Hadamard variations of domains method and are able to compute the shape derivative of $\lambda(\alpha)$ with respect to these deformations. See [5] for the details.

Here we follow a different path. Instead of considering regular perturbations we analyze the case of periodic oscillatory deformations where the amplitude of these oscillations converge to zero, and the period of these oscillations also converge to zero.

We start by describing the type of perturbations that we are to consider. Let $\Omega \subset \mathbb{R}^{n}$ be bounded. Assume that the boundary is regular $\left(C^{1}\right.$ will be enough for most of our arguments). For any $x_{0} \in \partial \Omega$ take $U \subset \mathbb{R}^{n}$ a neighborhood of $x_{0}$ and $\Phi: U^{\prime} \subset \mathbb{R}^{n-1} \rightarrow \mathbb{R}$, where $U^{\prime}$ is open and connected, such that

$$
\begin{aligned}
\partial \Omega \cap U & =\left\{\left(x_{1}, x^{\prime}\right) \in \mathbb{R}^{n}: x^{\prime} \in U^{\prime}, x_{1}=\Phi\left(x^{\prime}\right)\right\}, \\
\Omega \cap U & =\left\{\left(x_{1}, x^{\prime}\right) \in \mathbb{R}^{n}: x^{\prime} \in U^{\prime}, x_{1}<\Phi\left(x^{\prime}\right)\right\} .
\end{aligned}
$$

That is, we describe locally the boundary of $\Omega$ by the graph of a smooth function $\Phi$.

In each of this neighborhoods we assume that the perturbation is given by a $C^{1}$ periodic function as follows. Let $f: \mathbb{R}^{n-1} \rightarrow \mathbb{R}$ be a $C^{1}$ function, periodic with period $Y^{\prime}=[0,1]^{n-1}$.

Then the domains $\Omega_{\varepsilon}$ are given (locally) by

$$
\Omega_{\varepsilon} \cap U=\left\{\left(x_{1}, x^{\prime}\right) \in U: x^{\prime} \in U^{\prime}, x_{1}<\Phi\left(x^{\prime}\right)+\varepsilon^{a} f\left(\frac{x^{\prime}}{\varepsilon}\right)\right\}
$$




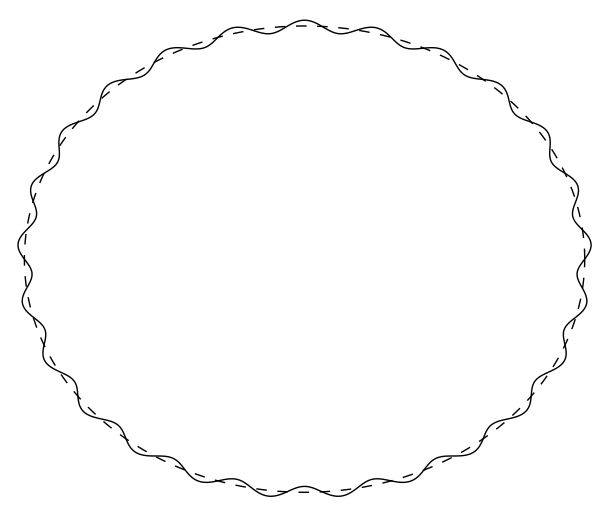

Figure 1. The unperturbed domain $\Omega$ in dashed line and the perturbed domain $\Omega_{\varepsilon}$ in solid line.

and therefore,

$$
\partial \Omega_{\varepsilon} \cap U=\left\{\left(x_{1}, x^{\prime}\right) \in \mathbb{R}^{N}: x^{\prime} \in U^{\prime}, x_{1}=\Phi\left(x^{\prime}\right)+\varepsilon^{a} f\left(\frac{x^{\prime}}{\varepsilon}\right)\right\} .
$$

See Figure 1.

By the results of [5], for every constant $\lambda_{\varepsilon}(\alpha)$, there exists an optimal window $\Gamma_{\varepsilon}$ and the corresponding eigenfunction $u_{\varepsilon} \in W^{1, p}\left(\Omega_{\varepsilon}\right)$ verifies that $\Gamma_{\varepsilon}=\left\{u_{\varepsilon}=\right.$ $0\} \cap \partial \Omega_{\varepsilon}$. Our goal is to study the behavior of these optimal windows $\Gamma_{\varepsilon}$, their eigenfunctions $u_{\varepsilon}$ and of the constants $\lambda\left(\Gamma_{\varepsilon}\right)=\lambda_{\varepsilon}(\alpha)$ when $\varepsilon \downarrow 0$.

Observe that these domains $\Omega_{\varepsilon}$ converge to $\Omega$ in practically any reasonable notion of set convergence in $\mathbb{R}^{n}$ (for instance in the Hausdorff complementary topology, the $L^{1}$ norm of the characteristic functions, etc.).

As we mentioned in the introduction, the behavior strongly depends on the amplitude of the oscillations measured in terms of the parameter $a>0$.

Three cases appear:

- The subcritical case, that corresponds to large oscillations with respect to the period $(a<1)$.

- The supercritical case, that corresponds to small oscillations with respect to the period $(a>1)$.

- The critical case, that corresponds to the case where amplitude and oscillations are of the same order $(a=1)$.

In the subcritical case, being the oscillations so big, the problem degenerates and the immersion is lost in the limit. This is a fattening phenomena of the boundary and it is reflected in the fact that the constants $\lambda\left(\Gamma_{\varepsilon}\right)$ converge to zero.

In the supercritical case, the oscillations are too small. Then, for small values of $\varepsilon$ the oscillations become imperceptible and that is reflected in the fact that the problem converges to the unperturbed one when $\varepsilon \downarrow 0$. 
Finally, the critical case is the most interesting. In this case, the oscillations and the periods are balanced and an homogeneization phenomena appears at the boundary. This homogenization is reflected in the appearance of a strange term at the boundary for the limit problem in the spirit of Cioranescu-Murat [3]. This phenomena have been observed in the work [8] where the pure eigenvalue problem is addressed.

Taking into account the above perturbation of the domain $\Omega$ we get the result.

Theorem 1.1. Let $\Omega \subset \mathbb{R}^{n}$ be an open, bounded set and assume that $\partial \Omega$ is of class $C^{1}$. Let $\left\{\Omega_{\varepsilon}\right\}_{\varepsilon>0}$ be the family of perturbed domains as described in (1.2). Let $\lambda_{\varepsilon}(\alpha)(0<\alpha<1)$ be the best Sobolev trace constant on $\Omega_{\varepsilon}$ given by (1.1) in the domain $\Omega_{\varepsilon}$.

Then the following statements hold true:

(1) (Subcritical case) If $a<1$ then $\lim _{\varepsilon \rightarrow 0} \lambda_{\varepsilon}(\alpha)=0$, moreover, we have the following asymptotic behavior

$$
\lambda_{\varepsilon}(\alpha) \leq C \varepsilon^{1-a}
$$

where the constant $C$ depends only on the function $f$ used in the perturbation.

(2) (Supercritical case) If $a>1$ then $\lim _{\varepsilon \rightarrow 0} \lambda_{\varepsilon}(\alpha)=\lambda(\alpha)$.

(3) (Critical case) If $a=1$ then $\lim _{\varepsilon \rightarrow 0} \lambda_{\varepsilon}(\alpha)=\lambda^{*}(\alpha)$, where $\lambda^{*}(\alpha)$ is defined as

$$
\begin{array}{r}
\lambda^{*}(\alpha):=\inf \left\{\frac{\int_{\Omega}|\nabla u|^{p}+|u|^{p} d x}{\int_{\partial \Omega}|u|^{p} d \mu^{*}}: u \in W^{1, p}(\Omega), \mu^{*}(\{u=0\} \cap \partial \Omega) \geq \alpha \mu^{*}(\partial \Omega)\right\}, \\
\text { and the measure } \mu^{*} \text { is given by } d \mu^{*}=m d S \text { with the weight } m \text { defined by }
\end{array}
$$

$$
m(x)=\frac{\int_{Y} \sqrt{1+\left|\nabla \Phi\left(x^{\prime}\right)+\nabla f(y)\right|^{2}} d y}{\sqrt{1+\left|\nabla \Phi\left(x^{\prime}\right)\right|^{2}}} .
$$

Nevertheless, our method is far more general and we are able to treat general perturbations where the periodic perturbation described above is just an (important) example. See Theorem 4.1 below. In particular, the perturbations considered here also cover the regular deformations considered in [5].

Moreover, we go further and analyze the behavior of these optimal windows $\Gamma_{\varepsilon}$ and of their corresponding eigenfunctions $u_{\varepsilon}$ as $\varepsilon \downarrow 0$. We found that, in the critical and in the subcritical case (an also in the more general framework of Theorem 4.1) these optimal windows converge (in a suitable sense) to an optimal window of the corresponding limit problem and also the convergence of their eigenfunctions to the eigenfunction of the limit problem. See Theorem 5.1.

1.2. Organization of the paper. After this introduction, the paper is organized as follows. In Section 2, we study the qualitative properties of the change of variables that deforms the original domain $\Omega$ into the periodically perturbed one $\Omega_{\varepsilon}$. In Section 3 we analyze the subcritical perturbation $(a<1)$ in Theorem 1.1. In Section 4 we prove one of the main theorems of the paper (Theorem 4.1) that implies, for instance, the critical $(a=1)$ and the supercritical $(a>1)$ cases in Theorem 1.1 and, moreover, the convergence of the corresponding eigenfunctions 
to the eigenfunction of the limit problem. Finally, in Section 5 we prove our second main theorem (Theorem 5.1) on the convergence of optimal windows.

\section{Estimates For the CHANGE OF VARIABLES}

In the analysis of the asymptotic behavior of the problem when $\varepsilon \downarrow 0$, it is of fundamental importance to understand the asymptotic behavior of the changes of variables that take the perturbed domains $\Omega_{\varepsilon}$ into $\Omega$.

Once these asymptotic behaviors are studied, the analysis is independent of the particular form of the change of variables and only depends on this asymptotic behavior.

Hence, given $\varepsilon>0$ we define the transformation $T_{\varepsilon}: \Omega_{\varepsilon} \rightarrow \Omega$ as

$$
\left(y_{1}, y^{\prime}\right)=T_{\varepsilon}\left(x_{1}, x^{\prime}\right)=\left(x_{1}-\varepsilon^{a} f\left(\frac{x^{\prime}}{\varepsilon}\right) \phi_{\varepsilon}(x), x^{\prime}\right),
$$

where, as usual, $x^{\prime}=\left(x_{2}, \ldots, x_{n}\right)$ and $\phi_{\varepsilon} \in C_{c}^{\infty}\left(\mathbb{R}^{n}\right)$ is supported on $B_{\sqrt{\varepsilon}}(\partial \Omega)=$ $\bigcup_{x \in \partial \Omega} B_{\sqrt{\varepsilon}}(x), \phi_{\varepsilon} \equiv 1$ in $\partial \Omega, 0 \leq \phi_{\varepsilon} \leq 1,\left|\nabla \phi_{\varepsilon}\right| \leq C \varepsilon^{-\frac{1}{2}}$.

We now compute the differential of $T_{\varepsilon}, D T_{\varepsilon}$.

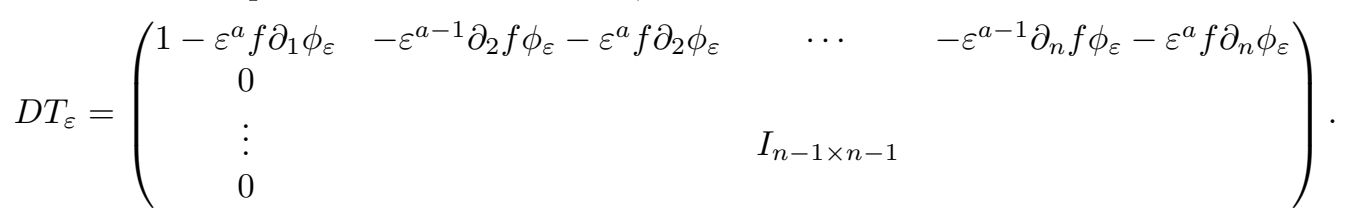

Observe that

$$
D T_{\varepsilon}(x)=I_{n \times n}-\varepsilon^{a} f\left(\frac{x^{\prime}}{\varepsilon}\right) A_{\varepsilon}(x)-\varepsilon^{a-1} \phi_{\varepsilon}(x) B\left(\frac{x^{\prime}}{\varepsilon}\right),
$$

where

$$
A_{\varepsilon}(x):=\left(\begin{array}{c}
\nabla \phi_{\varepsilon}(x) \\
0 \\
\vdots \\
0
\end{array}\right), \quad B\left(x^{\prime}\right):=\left(\begin{array}{cc}
0 & \nabla f\left(x^{\prime}\right) \\
0 & 0 \\
\vdots & \vdots \\
0 & 0
\end{array}\right)
$$

Finally, since $\|f\|_{\infty}<\infty$ and $\|\nabla f\|_{\infty}<\infty$ we have that

$$
\|B\|_{\infty}<\infty \text {. }
$$

Moreover, since $\left\|\nabla \phi_{\varepsilon}\right\|_{\infty} \leq C \varepsilon^{-\frac{1}{2}}$, we get

$$
\left\|A_{\varepsilon}\right\|_{\infty} \leq C \varepsilon^{-\frac{1}{2}} \chi_{\operatorname{supp}\left(\phi_{\varepsilon}\right)}
$$

and therefore we obtain that, calling $f_{\varepsilon}\left(x^{\prime}\right)=f\left(\frac{x^{\prime}}{\varepsilon}\right)$,

$$
\left\|\varepsilon^{a} f_{\varepsilon} A_{\varepsilon}\right\|_{\infty} \leq C \varepsilon^{a-\frac{1}{2}} \chi_{\operatorname{supp}\left(\phi_{\varepsilon}\right)} .
$$

On the other hand, calling $B_{\varepsilon}\left(x^{\prime}\right)=B\left(\frac{x^{\prime}}{\varepsilon}\right)$,

$$
\left\|\varepsilon^{a-1} \phi_{\varepsilon} B_{\varepsilon}\right\|_{\infty} \leq C \varepsilon^{a-1} \chi_{\operatorname{supp}\left(\phi_{\varepsilon}\right)} .
$$

Observe that when $a \geq 1$, we have that given $K \subset \Omega$ compact, $T_{\varepsilon}=i d_{\mathbb{R}^{n}}$ on $K$ for $\varepsilon>0$ small enough. In particular

$$
D T_{\varepsilon}=I_{n \times n} \quad \text { and } \quad J T_{\varepsilon}=1
$$


on $K$ for $\varepsilon>0$ small, where $J T_{\varepsilon}=\left|\operatorname{det}\left(D T_{\varepsilon}\right)\right|$ is the Jacobian of $T_{\varepsilon}$.

Finally, in the case $a>1, T_{\varepsilon} \rightarrow i d_{\mathbb{R}^{n}}$ in $C^{1}$ norm and, as a consequence, we get

$$
D T_{\varepsilon} \rightrightarrows I_{n \times n}, \quad J T_{\varepsilon} \rightrightarrows 1 \quad \text { and } \quad J_{\tau} T_{\varepsilon} \rightrightarrows 1,
$$

where $J_{\tau} T_{\varepsilon}=\left|D T_{\varepsilon}^{-1} \mathbf{n}\right| J T_{\varepsilon}$ is the tangential Jacobian of $T_{\varepsilon}, \mathbf{n}$ is the outer unit normal vector of $\Omega$ and “ $\rightrightarrows$ " means uniform convergence. See [10] for more details on the tangential Jacobian.

We need now to study the asymptotic behavior of the tangential Jacobian in the case $a=1$. In this case, for $x \in \partial \Omega$ taking into account that $\phi_{\varepsilon}=1$ on $\partial \Omega$ we get the following expression for the differential

$$
D T_{\varepsilon}(x)=I_{n \times n}-B\left(\frac{x^{\prime}}{\varepsilon}\right)+O\left(\varepsilon^{\frac{1}{2}}\right) .
$$

The following lemma gives the precise asymptotic behavior of the tangential Jacobian in this case.

Lemma 2.1. Given $g \in L^{1}(\partial \Omega)$ we have

$$
\int_{\partial \Omega} g J_{\tau} T_{\varepsilon}^{-1} d S \rightarrow \int_{\partial \Omega} g m d S, \text { when } \varepsilon \rightarrow 0 .
$$

That is $J_{\tau} T_{\varepsilon}^{-1} \stackrel{*}{\rightarrow} m$ weakly-* in $L^{\infty}(\partial \Omega)$, where $m$ is the function defined by (1.5).

Proof. Let $g \in C(\partial \Omega)$ be arbitrary. We first analyze the convergence locally, so we recall the construction of the perturbations. Then, let $U \subset \mathbb{R}^{n}$ be as in (1.2) and assume that $\operatorname{supp}(g) \subset U$. We then have that

$$
\begin{aligned}
\int_{\partial \Omega \cap U} g J_{\tau} T_{\varepsilon}^{-1} d S & =\int_{\partial \Omega_{\varepsilon} \cap U}\left(g \circ T_{\varepsilon}\right) d S \\
& =\int_{U^{\prime}}\left(g \circ T_{\varepsilon}\right)\left(x^{\prime}\right) \sqrt{1+\left|\nabla \Phi\left(x^{\prime}\right)+\nabla f\left(\frac{x^{\prime}}{\varepsilon}\right)\right|^{2}} d x^{\prime} .
\end{aligned}
$$

But now

$\int_{U^{\prime}}\left(g \circ T_{\varepsilon}\right)\left(x^{\prime}\right) \sqrt{1+\left|\nabla \Phi\left(x^{\prime}\right)+\nabla f\left(\frac{x^{\prime}}{\varepsilon}\right)\right|^{2}} d x^{\prime}=\int_{U^{\prime}}\left(g \circ T_{\varepsilon}\right)\left(x^{\prime}\right) m_{\varepsilon}\left(x^{\prime}\right) \sqrt{1+\left|\nabla \Phi\left(x^{\prime}\right)\right|^{2}} d x^{\prime}$, where

$$
m_{\varepsilon}\left(x^{\prime}\right):=m\left(x^{\prime}, \frac{x^{\prime}}{\varepsilon}\right), \quad m\left(x^{\prime}, y\right):=\frac{\sqrt{1+\left|\nabla \Phi\left(x^{\prime}\right)+\nabla f(y)\right|^{2}}}{\sqrt{1+\left|\nabla \Phi\left(x^{\prime}\right)\right|^{2}}} .
$$

Using that $f$ is periodic with period $Y$, it follows that $m\left(x^{\prime}, y\right)$ is periodic in $y$ with period $Y$ and hence

$$
m_{\varepsilon} \stackrel{*}{\rightarrow} m \quad \text { weakly-* in } L^{\infty}\left(\mathbb{R}^{n-1}\right) .
$$

See [1].

On the other hand, since $T_{\varepsilon} \rightrightarrows i d_{\mathbb{R}^{n}}$ it follows that $\left(g \circ T_{\varepsilon}\right) \rightrightarrows g$ uniformly on compact sets, in particular, $\left(g \circ T_{\varepsilon}\right) \rightarrow g$ in $L^{1}\left(U^{\prime}\right)$.

Combining all these facts, we arrive at

$$
\int_{U^{\prime}}\left(g \circ T_{\varepsilon}\right) m_{\varepsilon} \sqrt{1+|\nabla \Phi|^{2}} d x^{\prime} \rightarrow \int_{U^{\prime}} g m \sqrt{1+|\nabla \Phi|^{2}} d x^{\prime}=\int_{\partial \Omega} g m d S .
$$

The case where $g \in C(\partial \Omega)$ is arbitrary, follows by a standard arguments using the partition of unity and is omitted. 
Finally, if $g \in L^{1}(\partial \Omega)$ a standard approximation argument gives the desired result.

Summing up we have proved the following result for the perturbation (2.1).

Theorem 2.2. Let $\left\{T_{\varepsilon}\right\}_{\varepsilon>0}$ be the transformation given by (2.1). Then the following estimates hold:

(1) If $a>1, T_{\varepsilon} \rightarrow i d_{\mathbb{R}^{n}}$ in $C^{1}$ norm as $\varepsilon \rightarrow 0$. In consequence

$$
T_{\varepsilon} \rightrightarrows i d_{\mathbb{R}^{n}}, D T_{\varepsilon} \rightrightarrows I_{n \times n}, J T_{\varepsilon} \rightrightarrows 1 \text { and } J_{\tau} T_{\varepsilon} \rightrightarrows 1 \text {. }
$$

(2) If $a=1$, we have that for any compact set $K \subset \Omega$ there exists $\varepsilon_{0}>0$ such that

$$
\left.T_{\varepsilon}\right|_{K}=i d_{K}
$$

for every $0<\varepsilon<\varepsilon_{0}$. Moreover,

$$
J_{\tau} T_{\varepsilon}^{-1} \stackrel{*}{\rightarrow} m \quad \text { weakly- * in } L^{\infty}(\partial \Omega),
$$

where $m$ is the function given by (1.5).

\section{Subcritical CASE $(a<1)$}

In this section we prove the result in the subcritical case. This is the simplest of the three cases.

Proof. Let $\alpha \in(0,1)$ and let us take $\Gamma_{0} \subset \partial \Omega$ as the closure of a relative open and connected set such that $\left|\Gamma_{0}\right|_{n-1}>\alpha|\partial \Omega|_{n-1}$.

Given $\delta>0$, consider the sets $U_{\delta}=B_{\delta}\left(\Gamma_{0}\right)$ defined as

$$
U_{\delta}:=\left\{x \in \mathbb{R}^{n}: \operatorname{dist}\left(x, \Gamma_{0}\right)<\delta\right\}
$$

and take $\Gamma_{1} \subset \partial \Omega \backslash \bar{U}_{2 \delta}$ such that $\left|\Gamma_{1}\right|_{n-1}>0$.

Let now $\phi \in C^{1}(\bar{\Omega})$ be such that $\phi \equiv 0$ in $U_{\delta}, \phi \equiv 1$ in $\Omega \backslash U_{2 \delta}$ and $0 \leq \phi \leq 1$, $|\nabla \phi| \leq C \delta^{-1}$ in $U_{2 \delta} \backslash U_{\delta}$.

Observe that if we denote by $\Gamma_{0, \varepsilon} \subset \partial \Omega_{\varepsilon}$ to the portion of the boundary of $\Omega_{\varepsilon}$ that comes from perturbing $\Gamma_{0}$, one has that $\phi \equiv 0$ in $\Gamma_{0, \varepsilon}$ for every $\varepsilon>0$ small. Moreover, is easy to see that $\left|\Gamma_{0, \varepsilon}\right|_{n-1} \geq \alpha\left|\partial \Omega_{\varepsilon}\right|_{n-1}$. Then, $\phi$ is admissible in the characterization of $\lambda\left(\Gamma_{0, \varepsilon}\right)$. As a consequence, we get the following estimate:

$$
\lambda_{\varepsilon}(\alpha) \leq \lambda\left(\Gamma_{0, \varepsilon}\right) \leq \frac{\int_{\Omega_{\varepsilon}}|\nabla \phi|^{p}+|\phi|^{p} d x}{\int_{\partial \Omega_{\varepsilon}}|\phi|^{p} d S} .
$$

This quotient can be easily estimated. In fact,

$$
\int_{\Omega_{\varepsilon}}|\nabla \phi|^{p} d x \leq C\left|\Omega_{\varepsilon}\right|_{n}, \quad \int_{\Omega_{\varepsilon}}|\phi|^{p} d x \leq\left|\Omega_{\varepsilon}\right|_{n}
$$

with $C=C(\delta)$.

On the other hand,

$$
\int_{\partial \Omega_{\varepsilon}}|\phi|^{p} d S \geq \int_{\partial \Omega_{\varepsilon} \backslash \bar{U}_{2 \delta}}|\phi|^{p} d S=\left|\partial \Omega_{\varepsilon} \backslash \bar{U}_{2 \delta}\right|_{n-1} \geq\left|\Gamma_{1, \varepsilon}\right|_{n-1},
$$

where $\Gamma_{1, \varepsilon}$ stands for the perturbed set obtained from $\Gamma_{1} \subset \partial \Omega \backslash \bar{U}_{2 \delta}$. 
But,

$$
\begin{aligned}
\left|\Gamma_{1, \varepsilon}\right|_{n-1} & =\int_{U^{\prime}} \sqrt{1+\left|\nabla \Phi\left(x^{\prime}\right)+\varepsilon^{a-1} \nabla f\left(\frac{x^{\prime}}{\varepsilon}\right)\right|^{2}} d x^{\prime} \\
& =\varepsilon^{a-1} \int_{U^{\prime}} \sqrt{\varepsilon^{2(1-a)}+\left|\varepsilon^{1-a} \nabla \Phi\left(x^{\prime}\right)+\nabla f\left(\frac{x^{\prime}}{\varepsilon}\right)\right|^{2}} d x^{\prime} .
\end{aligned}
$$

Let us now estimate this last integral.

$$
\begin{aligned}
\int_{U^{\prime}} & \sqrt{\varepsilon^{2(1-a)}+\left|\varepsilon^{1-a} \nabla \Phi\left(x^{\prime}\right)+\nabla f\left(\frac{x^{\prime}}{\varepsilon}\right)\right|^{2}} d x^{\prime} \\
= & \int_{U^{\prime}}\left(\sqrt{\varepsilon^{2(1-a)}+\left|\varepsilon^{1-a} \nabla \Phi\left(x^{\prime}\right)+\nabla f\left(\frac{x^{\prime}}{\varepsilon}\right)\right|^{2}}-\left|\nabla f\left(\frac{x^{\prime}}{\varepsilon}\right)\right|\right)+\left|\nabla f\left(\frac{x^{\prime}}{\varepsilon}\right)\right| d x^{\prime} .
\end{aligned}
$$

If we now denote by $\rho_{\varepsilon}\left(x^{\prime}\right)=\sqrt{\varepsilon^{2(1-a)}+\left|\varepsilon^{1-a} \nabla \Phi\left(x^{\prime}\right)+\nabla f\left(\frac{x^{\prime}}{\varepsilon}\right)\right|^{2}}-\left|\nabla f\left(\frac{x^{\prime}}{\varepsilon}\right)\right|$, it is not difficult to see that $\left|\rho_{\varepsilon}\left(x^{\prime}\right)\right| \leq \varepsilon^{1-a}\left(1+\left|\nabla \Phi\left(x^{\prime}\right)\right|\right)$, from where it follows that

$$
\int_{U^{\prime}}\left(\sqrt{\varepsilon^{2(1-a)}+\left|\varepsilon^{1-a} \nabla \Phi\left(x^{\prime}\right)+\nabla f\left(\frac{x^{\prime}}{\varepsilon}\right)\right|^{2}}-\left|\nabla f\left(\frac{x^{\prime}}{\varepsilon}\right)\right|\right) d x^{\prime} \rightarrow 0 \quad \text { when } \varepsilon \rightarrow 0 .
$$

Finally, by the periodicity of $f$, we conclude that

$$
\int_{U^{\prime}}\left|\nabla f\left(\frac{x^{\prime}}{\varepsilon}\right)\right| d x^{\prime} \rightarrow \int_{Y}|\nabla f(y)| d y=: \overline{|\nabla f|}>0 .
$$

These estimates allow us to conclude that,

$$
\left|\Gamma_{1, \varepsilon}\right|_{n-1} \geq \varepsilon^{a-1} \frac{\overline{|\nabla f|}}{2},
$$

for every $\varepsilon>0$ small.

Now, from (3.1), (3.2) and (3.3), we obtain

$$
\lambda_{\varepsilon}(\alpha) \leq C \varepsilon^{1-a} \rightarrow 0 \quad \text { when } \varepsilon \rightarrow 0
$$

as we wanted to show.

\section{Supercritical And CRitical Cases $(a \leq 1)$}

Now taking into account Theorem 2.2, we note that the supercritical and critical cases in Theorem 1.1 are special cases of a more general result.

Indeed if $T_{\varepsilon}: \Omega_{\varepsilon} \rightarrow \Omega$ is a family of perturbations which satisfies the following condition:

$$
\begin{cases}T_{\varepsilon}=i d_{\mathbb{R}^{n}} . & \text { on each compact set } K \subset \Omega \text { for } \varepsilon<\varepsilon_{0}(K) \\ J_{\tau} T_{\varepsilon}^{-1} \stackrel{*}{\rightarrow} m, & \text { weakly* in } L^{\infty}(\partial \Omega) \text { when } \varepsilon \rightarrow 0,\end{cases}
$$

where $m \in L^{\infty}(\partial \Omega)$ then we get the following general result. 
Theorem 4.1. Let $\left\{T_{\varepsilon}\right\}_{\varepsilon>0}$ be a family of perturbations that satisfies condition (4.1). Then

$$
\lambda_{\varepsilon}(\alpha) \rightarrow \lambda^{*}(\alpha) \text {, when } \varepsilon \rightarrow 0,
$$

where $\lambda_{\varepsilon}(\alpha)$ is given by (1.1) on $\Omega_{\varepsilon}$ and $\lambda^{*}(\alpha)$ is given by

$$
\lambda^{*}(\alpha)=\inf \left\{\frac{\int_{\Omega}|\nabla u|^{p}+|u|^{p} d x}{\int_{\partial \Omega}|u|^{p} d \mu^{*}}: u \in W^{1, p}(\Omega), \mu^{*}(\{u=0\} \cap \partial \Omega) \geq \alpha \mu^{*}(\partial \Omega)\right\} .
$$

Here the measure $\mu^{*}$ is given by $d \mu^{*}=m d S$.

Moreover, if $u_{\varepsilon}$ is an eigenfunction associated to $\lambda_{\varepsilon}(\alpha)$ normalized as $\left\|u_{\varepsilon}\right\|_{L^{p}\left(\partial \Omega_{\varepsilon}\right)}=$ 1 , then the sequence $\left\{u_{\varepsilon} \circ T_{\varepsilon}^{-1}\right\}_{\varepsilon>0} \subset W^{1, p}(\Omega)$ is weakly pre compact and every accumulation point is an eigenfunction of $\lambda^{*}(\alpha)$.

Clearly, Theorem 4.1 implies the critical $(a=1)$ and supercritical $(a>1)$ cases in Theorem 1.1. Also Theorem 4.1 implies Theorem 6.2 in [5].

Before starting the proof we need the following observations.

Let $\Omega_{1}, \Omega_{2} \subset \mathbb{R}^{n}$ be open domains and suppose that there exists a diffeomorphism $T: \Omega_{1} \rightarrow \Omega_{2}$. This diffeomorphism $T$ induces the mapping

$$
\mathcal{T}: W^{1, p}\left(\Omega_{2}\right) \rightarrow W^{1, p}\left(\Omega_{1}\right), \quad \mathcal{T}(u)=u \circ T .
$$

This mapping is linear, continuous and invertible, with $\mathcal{T}^{-1} v=v \circ T^{-1}$. Moreover a direct application of the Change of Variables Theorem implies that

$$
\int_{\Omega_{1}}|\mathcal{T} u|^{p} d x \leq\left\|J T^{-1}\right\|_{\infty} \int_{\Omega_{2}}|u|^{p} d x
$$

and

$$
\int_{\Omega_{1}}|\nabla(\mathcal{T} u)|^{p} d x \leq\left\|J T^{-1}\right\|_{\infty}\|D T\|_{\infty} \int_{\Omega_{2}}|\nabla u|^{p} d y
$$

Then if we consider now the general pertubations $T_{\varepsilon}: \Omega_{\varepsilon} \rightarrow \Omega$ which satisfies the properties (4.1) we get the associated mappings $\mathcal{T}_{\varepsilon}: W^{1, p}(\Omega) \rightarrow W^{1, p}\left(\Omega_{\varepsilon}\right)$, which are linear, invertible and, by (4.3) and (4.4), bi-continuous.

With this in mind we define the functions $Q_{\varepsilon}: W^{1, p}\left(\Omega_{\varepsilon}\right) \rightarrow \mathbb{R}, Q: W^{1, p}(\Omega) \rightarrow \mathbb{R}$ by

$$
Q_{\varepsilon}(u)=\int_{\Omega_{\varepsilon}}|\nabla u|^{p}+|u|^{p} d x
$$

and

$$
Q(v)=\int_{\Omega}|\nabla v|^{p}+|v|^{p} d y .
$$

We now consider the function $\tilde{Q}_{\varepsilon}: W^{1, p}(\Omega) \rightarrow \mathbb{R}$ defined by $\tilde{Q}_{\varepsilon}=Q_{\varepsilon} \circ \mathcal{T}_{\varepsilon}$.

We introduce the sets

$$
X_{\alpha}^{\varepsilon}:=\left\{u \in W^{1, p}\left(\Omega_{\varepsilon}\right):\left|\{u=0\} \cap \partial \Omega_{\varepsilon}\right|_{n-1} \geq \alpha\left|\partial \Omega_{\varepsilon}\right|_{n-1} \text { and }\left\|u_{\varepsilon}\right\|_{L^{p}\left(\partial \Omega_{\varepsilon}\right)}=1\right\},
$$

$$
\tilde{X}_{\alpha}^{\varepsilon}:=\mathcal{T}_{\varepsilon}\left(X_{\alpha}^{\varepsilon}\right)=\left\{v \in W^{1, p}(\Omega): v \circ T_{\varepsilon} \in X_{\alpha}^{\varepsilon}\right\},
$$

$$
X_{\alpha}^{*}:=\left\{v \in W^{1, p}(\Omega): \mu^{*}(\{v=0\} \cap \partial \Omega) \geq \alpha \mu^{*}(\partial \Omega) \text { and }\|v\|_{L^{p}\left(d \mu^{*}\right)}=1\right\},
$$


where $d \mu^{*}=m d S$.

With the above notations, we can write

$$
\lambda_{\varepsilon}(\alpha)=\inf _{u \in X_{\alpha}^{\varepsilon}} Q_{\varepsilon}(u)=\inf _{v \in \tilde{X}_{\alpha}^{\varepsilon}} \tilde{Q}_{\varepsilon}(v) \quad \text { and } \quad \lambda^{*}(\alpha)=\inf _{v \in X_{\alpha}^{*}} Q(v) .
$$

In order to prove the convergence of these minima, we use the notion of $\Gamma-$ convergence. This notion was introduced by E. De Giorgi in the 60 's and is by now a classical subject in dealing with variational problems. We refer the reader to the books of A. Braides [2] and of G. Dal Maso [4].

For the sake of completeness, we recall the definition of $\Gamma$-convergence.

Definition 4.2. Let $(X, d)$ be a metric space and let $J_{\varepsilon}, J: X \rightarrow(-\infty,+\infty]$. We say that $J_{\varepsilon} \Gamma$-converges to $J$ as $\varepsilon \rightarrow 0$ if

- (liminf inequality) For every $x \in X$ and for every sequence $\left\{x_{\varepsilon}\right\}_{\varepsilon>0} \subset X$ such that $x_{\varepsilon} \rightarrow x$, we have

$$
J(x) \leq \liminf _{\varepsilon \rightarrow 0} J_{\varepsilon}\left(x_{\varepsilon}\right) .
$$

- (limsup inequality) For every $x \in X$ there exists $\left\{y_{\varepsilon}\right\}_{\varepsilon>0} \subset X$ such that $y_{\varepsilon} \rightarrow x$ and

$$
J(x) \geq \limsup _{\varepsilon \rightarrow 0} J_{\varepsilon}\left(y_{\varepsilon}\right) .
$$

We denote this convergence by $J=\Gamma-\lim _{\varepsilon \rightarrow 0} J_{\varepsilon}$.

This notion is extremely useful in dealing with convergence of minima as the following theorem shows.

Theorem 4.3. Let $(X, d)$ be a metric space and let $J_{\varepsilon}, J: X \rightarrow(-\infty,+\infty]$ be such that $J=\Gamma-\lim _{\varepsilon \rightarrow 0} J_{\varepsilon}$. Assume that for every $\varepsilon>0$ there exists $x_{\varepsilon} \in X$ such that $J_{\varepsilon}\left(x_{\varepsilon}\right)=\inf _{X} J_{\varepsilon}$. Moreover, assume that $\left\{x_{\varepsilon}\right\}_{\varepsilon>0}$ is precompact in $X$. Then

- $\inf _{X} J=\lim _{\varepsilon \rightarrow 0} \inf _{X} J_{\varepsilon}$.

- If $x$ is any accumulation point of the sequence $\left\{x_{\varepsilon}\right\}_{\varepsilon>0}$, then $J(x)=\inf _{X} J$.

The proof of this theorem is elementary and can be found in any of the above mentioned books $[2,4]$.

We apply this theorem to the functions $J_{\varepsilon}, J: L^{p}(\Omega) \rightarrow(-\infty,+\infty]$ given by

$$
\begin{aligned}
& J_{\varepsilon}(v):= \begin{cases}\tilde{Q}_{\varepsilon}(v) & \text { if } v \in \tilde{X}_{\alpha}^{\varepsilon} \\
+\infty & \text { if not, }\end{cases} \\
& J(v):= \begin{cases}Q(v) & \text { if } v \in X_{\alpha}^{*} \\
+\infty & \text { if not. }\end{cases}
\end{aligned}
$$

We begin by showing the $\Gamma$-convergence of the functionals. For this we need the following lemmas.

Lemma 4.4. Let $(X, \Sigma, \nu)$ be a measure space of finite measure and let $\left\{f_{k}\right\}_{k \in \mathbb{N}}, f$ be $\Sigma$-measurable and nonnegative functions such that $f_{k} \rightarrow f \nu$-a.e. 
Let $\left\{\mu_{k}\right\}_{k \in \mathbb{N}}$ and $\mu$ be absolutely continuous measures with respect to $\nu$ such that $\mu_{k}(A) \rightarrow \mu(A)$, for every $A \in \Sigma$.

Then

$$
\limsup _{k \rightarrow \infty} \mu_{k}\left(\left\{f_{k}=0\right\}\right) \leq \mu(\{f=0\}) .
$$

Remark 4.5. When $\mu_{k}=\mu$ for every $k \in \mathbb{N}$ this is well known with a simple proof. In this case, the difficulty appears since the measures vary. We do not know if this result is known nor if the hypotheses are optimal. Nevertheless it will suffices for our purposes.

Remark 4.6. By standard arguments, it can be shown that the condition $\mu_{k}(A) \rightarrow$ $\mu(A)$ for every $A \in \Sigma$ is equivalent to the weak convergence of the densities of the measures in $L^{1}(X, \nu)$.

Proof of Lemma 4.4. Assume by contradiction that there exists $\delta>0$ such that, for all $k_{0} \in \mathbb{N}$ there exists $k \geq k_{0}$ such that

$$
\mu(\{f=0\})+\delta<\mu_{k}\left(\left\{f_{k}=0\right\}\right) .
$$

Since $\{f=0\}=\bigcap_{j=1}^{\infty}\left\{f \leq \frac{1}{j}\right\}$ it follows that $\mu(\{f=0\})=\lim _{j \rightarrow \infty} \mu\left(\left\{f \leq \frac{1}{j}\right\}\right)$. Hence, there exists $j_{0} \in \mathbb{N}$ such that, for $j \geq j_{0}$,

so

$$
\begin{gathered}
\mu\left(\left\{f \leq \frac{1}{j}\right\}\right)+\frac{\delta}{2}<\mu_{k}\left(\left\{f_{k}=0\right\}\right) . \\
\left\{f \leq \frac{1}{j}\right\} \supset \bigcap_{k_{0}=1}^{\infty} \bigcup_{k \geq k_{0}}\left\{f_{k}<\frac{1}{j}\right\},
\end{gathered}
$$

$$
\mu\left(\bigcap_{k_{0}=1}^{\infty} \bigcup_{k \geq k_{0}}\left\{f_{k}<\frac{1}{j}\right\}\right) \leq \mu\left(\left\{f \leq \frac{1}{j}\right\}\right)
$$

But, since

$$
\lim _{k_{0} \rightarrow \infty} \mu\left(\bigcup_{k \geq k_{0}}\left\{f_{k}>\frac{1}{j}\right\}\right)=\mu\left(\bigcap_{k_{0}=1}^{\infty} \bigcup_{k \geq k_{0}}\left\{f_{k}<\frac{1}{j}\right\}\right),
$$

there exists $k_{0}(\delta)$ such that

$$
\mu\left(\bigcup_{k \geq k_{0}}\left\{f_{k}<\frac{1}{j}\right\}\right)+\frac{\delta}{4}<\mu_{k}\left(\left\{f_{k}=0\right\}\right) .
$$

Calling $A=\cup_{i \geq k_{0}}\left\{f_{i}<\frac{1}{j}\right\}$, by hypothesis we have that $\lim _{k \rightarrow \infty} \mu_{k}(A)=\mu(A)$ and therefore,

$$
\mu_{k}\left(\bigcup_{i \geq n_{0}}\left\{f_{i}<\frac{1}{j}\right\}\right)+\frac{\delta}{8}<\mu_{k}\left(\left\{f_{k}=0\right\}\right) .
$$

Finally observe that $\left\{f_{k}>\frac{1}{j}\right\} \subset \bigcup_{i \geq n_{0}}\left\{f_{i}<\frac{1}{j}\right\}$ and hence we can conclude that

$$
\mu_{k}\left(\left\{f_{k}<\frac{1}{j}\right\}\right)+\frac{\delta}{8}<\mu_{k}\left(\left\{f_{k}=0\right\}\right),
$$

a contradiction. 
Lemma 4.7. Let $\tilde{X}_{\alpha}^{\varepsilon}, X_{\alpha}^{*} \subset W^{1, p}(\Omega)$ be the sets defined in (4.8) and (4.9) respectively. Then, given $v_{\varepsilon} \in \tilde{X}_{\alpha}^{\varepsilon}$ such that $v_{\varepsilon} \rightarrow v$ weakly in $W^{1, p}(\Omega)$, it follows that $v \in X_{\alpha}^{*}$.

Reciprocally, for every $v \in X_{\alpha}^{*}$ there exists a sequence $\left\{\varepsilon_{k}\right\}_{k \in \mathbb{N}}$ such that $\varepsilon_{k} \downarrow 0$ and $v_{k} \in \tilde{X}_{\alpha}^{\varepsilon_{k}}$ such that $v_{k} \rightarrow v$ weakly in $W^{1, p}(\Omega)$. Moreover, the sequence can be taken to converge strongly in $W^{1, p}(\Omega)$.

Remark 4.8. The result of the previous Lemma says that the sets $\tilde{X}_{\alpha}^{\varepsilon}$ converges in the sense of Mosco to the set $X_{\alpha}^{*}$. See [10].

Proof. Let $v \in X_{\alpha}^{*}$ and set $\Gamma=\{v=0\} \cap \partial \Omega$.

Given $k \in \mathbb{N}$ define $\tilde{v}_{k}:=\max \left\{v-\frac{1}{k}, 0\right\}$. Then, $\Gamma_{k}=\left\{\tilde{v}_{k}=0\right\} \cap \partial \Omega$ verifies that $\mu^{*}\left(\Gamma_{k}\right)>\mu^{*}(\Gamma)$ (recall that the weight $m$ is strictly positive). So, there exists $\rho_{k}>0$ such that

$$
\mu^{*}\left(\Gamma_{k}\right) \geq\left(1+\rho_{k}\right) \alpha \mu^{*}(\partial \Omega)
$$

It is straightforward to check that $\tilde{v}_{k} \rightarrow v$ strongly in $W^{1, p}(\Omega)$ as $k \rightarrow \infty$.

Now let $t_{k, \varepsilon}>0$ be such that $v_{k, \varepsilon}:=t_{k, \varepsilon} \tilde{v}_{k}$ verifies that $\left\|v_{k, \varepsilon} \circ T_{\varepsilon}\right\|_{L^{p}\left(\partial \Omega_{\varepsilon}\right)}=$ 1. It is easy to see that $\tilde{v}_{k} \rightarrow v(k \rightarrow \infty)$ strongly in $L^{p}(\partial \Omega)$ implies that $\lim _{\varepsilon \downarrow 0}\left(\lim _{k \rightarrow \infty} t_{k, \varepsilon}\right) \rightarrow 1$. So, we have that $v_{k, \varepsilon} \rightarrow v$ strongly in $W^{1, p}(\Omega)$ as $k \rightarrow \infty$ and $\varepsilon \downarrow 0$.

It remains to check that, given $k \in \mathbb{N}$ there exists $\varepsilon_{k}$ with $\varepsilon_{k} \downarrow 0$ such that $v_{k}:=v_{k, \varepsilon_{k}} \in \tilde{X}_{\alpha}^{\varepsilon_{k}}$ and for this we have only to check that

$$
\mu_{\varepsilon_{k}}\left(\Gamma_{k}\right) \geq \alpha \mu_{\varepsilon_{k}}(\partial \Omega)
$$

where $\Gamma_{k}=\left\{v_{k}=0\right\} \cap \partial \Omega=\left\{\tilde{v}_{k}=0\right\} \cap \partial \Omega$ and $d \mu_{\varepsilon}=J_{\tau} T_{\varepsilon} d S$.

But, since $\mu_{\varepsilon}(A) \rightarrow \mu^{*}(A)$ for every $d S$-measurable set $A \subset \partial \Omega$, we have that there exists $\varepsilon_{k}$ such that

$$
\mu_{\varepsilon_{k}}\left(\Gamma_{k}\right) \geq\left(1+\rho_{k}\right)^{-\frac{1}{2}} \mu^{*}\left(\Gamma_{k}\right) \quad \text { and } \quad \mu^{*}(\partial \Omega) \geq\left(1+\rho_{k}\right)^{-\frac{1}{2}} \mu_{\varepsilon_{k}}(\partial \Omega) .
$$

Combining (4.12) and (4.13) we arrive at

$$
\begin{aligned}
\mu_{\varepsilon_{k}}\left(\Gamma_{k}\right) & \geq\left(1+\rho_{k}\right)^{-\frac{1}{2}} \mu^{*}\left(\Gamma_{k}\right) \\
& \geq\left(1+\rho_{k}\right)^{\frac{1}{2}} \alpha \mu^{*}(\partial \Omega) \\
& \geq \alpha \mu_{\varepsilon_{k}}(\partial \Omega) .
\end{aligned}
$$

Now, we need to see that if $v_{\varepsilon} \in \tilde{X}_{\alpha}^{\varepsilon}$ is such that $v_{\varepsilon} \rightarrow v$, then $v \in X_{\alpha}^{*}$. But this is an immediate consequence of Lemma 4.4 .

In fact, Lemma 4.4 is applied to the functions $v_{\varepsilon}, v \in W^{1, p}(\Omega) \subset L^{p}(\partial \Omega)$ (recall that we can assume that $v_{\varepsilon} \rightarrow v d S$-a.e.on $\partial \Omega$ ) and the measures

$$
d \mu_{\varepsilon}=J_{\tau} T_{\varepsilon}^{-1} d S, \quad d \mu^{*}=m d S, \quad d \nu=d S .
$$


As a consequence, we get

$$
\begin{aligned}
\mu^{*}(\{v=0\} \cap \partial \Omega) & \geq \limsup _{\varepsilon \rightarrow 0} \mu_{\varepsilon}\left(\left\{v_{\varepsilon}=0\right\} \cap \partial \Omega\right)=\limsup _{\varepsilon \rightarrow 0} \int_{\left\{v_{\varepsilon}=0\right\} \cap \partial \Omega} J_{\tau} T_{\varepsilon}^{-1} d S \\
& =\limsup _{\varepsilon \rightarrow 0}\left|\left\{v_{\varepsilon} \circ T_{\varepsilon}=0\right\} \cap \partial \Omega_{\varepsilon}\right|_{n-1} \\
& \geq \limsup _{\varepsilon \rightarrow 0} \alpha\left|\partial \Omega_{\varepsilon}\right|_{n-1}=\alpha \mu^{*}(\partial \Omega) .
\end{aligned}
$$

This finishes the proof.

Unfortunately, we are not able to prove the $\Gamma$-convergence of the functionals in its full generality. In fact we can only prove $\Gamma$-convergence for the supercritical case, that in this general setting will be in the case where $T_{\varepsilon} \rightarrow i d_{\mathbb{R}^{n}}$ in the $C^{1}$ topology.

For the more general setting of (4.1), we can prove a weaker version of $\Gamma$-convergence under which Theorem 4.3 still holds. Namely

Proposition 4.9. Let $J_{\varepsilon}, J: L^{p}(\Omega) \rightarrow(-\infty,+\infty]$ be the functionals defined by (4.10)-(4.11). Assume that the transformations $T_{\varepsilon}$ verify (4.1). Then:

- for every sequence $\left\{v_{\varepsilon}\right\}_{\varepsilon>0} \subset L^{p}(\Omega)$ of minimizers of $\left\{J_{\varepsilon}\right\}_{\varepsilon>0}$ such that $v_{\varepsilon} \rightarrow v$ in $L^{p}(\Omega)$, we have that

$$
J(v) \leq \liminf _{\varepsilon \rightarrow 0} J_{\varepsilon}\left(v_{\varepsilon}\right) .
$$

- Moreover, for every $v \in L^{p}(\Omega)$, there exists $v_{k} \in L^{p}(\Omega)$ and $\varepsilon_{k} \downarrow 0$ such that $v_{k} \rightarrow v$ in $L^{p}(\Omega)$ and

$$
J(v) \geq \limsup _{k \rightarrow \infty} J_{\varepsilon_{k}}\left(v_{k}\right) .
$$

Remark 4.10. Observe that the only difference with respect to $\Gamma$-convergence is that we do not prove the liminf inequality for every sequence $\left\{v_{\varepsilon}\right\}_{\varepsilon>0}$, but only for sequences of minimizers. It is straightforward to check that the conclusions of Theorem 4.3 still hold under this weaker assumption.

Proof. We will divide the proof into two parts.

lim inf inequality: Let $v_{\varepsilon} \in \tilde{X}_{\alpha}^{\varepsilon}$ be such that $\tilde{Q}_{\varepsilon}\left(v_{\varepsilon}\right)=\inf _{\tilde{X}_{\alpha}^{\varepsilon}} \tilde{Q}_{\varepsilon}$ and assume that $v_{\varepsilon} \rightarrow v$ in $L^{p}(\Omega)$ as $\varepsilon \downarrow 0$ for some $v \in X_{\alpha}^{*}$. We can assume that

$$
\liminf _{\varepsilon \rightarrow 0} \tilde{Q}_{\varepsilon}\left(v_{\varepsilon}\right)<+\infty
$$

otherwise there is nothing to prove. It is immediate to see that (4.3) and (4.4) imply that

$$
\left\|v_{\varepsilon}\right\|_{W^{1, p}(\Omega)}=Q\left(v_{\varepsilon}\right) \leq C \tilde{Q}_{\varepsilon}\left(v_{\varepsilon}\right)
$$

and so, by (4.14) we conclude that $\left\{v_{\varepsilon}\right\}_{\varepsilon>0}$ is bounded on $W^{1, p}(\Omega)$ and since $v_{\varepsilon} \rightarrow v$ in $L^{p}(\Omega)$ it easily follows that $v_{\varepsilon} \rightarrow v$ weakly in $W^{1, p}(\Omega)$.

Since $v_{\varepsilon} \rightarrow v$ strongly in $L^{p}(\Omega)$ and $J T_{\varepsilon} \rightarrow 1$ a.e. in $\Omega$ and are uniformly bounded it follows that

$$
\int_{\Omega}\left|v_{\varepsilon}\right|^{p} J T_{\varepsilon}^{-1} d x \rightarrow \int_{\Omega}|v|^{p} d x, \text { as } \varepsilon \downarrow 0 .
$$


Taking into account the expressions of $\tilde{Q}_{\varepsilon}\left(v_{\varepsilon}\right)$ and $Q(v)$, it remains to show that

$$
\liminf _{\varepsilon \rightarrow 0} \int_{\Omega}\left|\nabla v_{\varepsilon}\left(D T_{\varepsilon} \circ T_{\varepsilon}^{-1}\right)\right|^{p} J T_{\varepsilon}^{-1} d x \geq \int_{\Omega}|\nabla v|^{p} d x .
$$

We first show that $\nabla v_{\varepsilon} \rightarrow \nabla v$ a.e. in $\Omega$. To this end we need the fact that the sequence $\left\{v_{\varepsilon}\right\}_{\varepsilon>0}$ is a sequence of minimizers for $\tilde{Q}_{\varepsilon}$ and therefore they verify the Euler-Lagrange equation associated to the functional $\tilde{Q}_{\varepsilon}$. That is

$$
\begin{aligned}
\int_{\Omega}\left(\left|\nabla v_{\varepsilon}\left(D T_{\varepsilon} \circ T_{\varepsilon}^{-1}\right)\right|^{p-2} \nabla v_{\varepsilon}\left(D T_{\varepsilon} \circ T_{\varepsilon}^{-1}\right) \cdot \nabla \psi+\right. & \left.\left|v_{\varepsilon}\right|^{p-2} v_{\varepsilon} \psi\right) J T_{\varepsilon}^{-1} d x \\
& =\lambda_{\varepsilon}(\alpha) \int_{\partial \Omega}\left|v_{\varepsilon}\right|^{p-2} v_{\varepsilon} \psi J_{\tau} T_{\varepsilon}^{-1} d S
\end{aligned}
$$

for every $\psi \in W_{\Gamma}^{1, p}(\Omega)$.

Take now $K \subset \Omega$ a compact set, let $\delta=\frac{1}{2} d(K, \partial \Omega)$ and write $K_{\delta}=\{x \in$ $\Omega: d(x, K)<\delta\}$. Therefore $K \subset K_{\delta} \subset \subset \Omega$ and if $\varepsilon$ is small enough, we have that $T_{\varepsilon}=i d_{\mathbb{R}^{n}}$ on $K_{\delta}$.

Observe now that if $\psi_{0} \in W_{\Gamma}^{1, p}(\Omega)$ is such that $\operatorname{supp}\left(\psi_{0}\right) \subset K_{\delta}$, then

$$
\int_{\Omega}\left|\nabla v_{\varepsilon}\right|^{p-2} \nabla v_{\varepsilon} \nabla \psi_{0}+\left|v_{\varepsilon}\right|^{p-2} v_{\varepsilon} \psi_{0} d x=0
$$

So consider $\eta \in C_{c}^{\infty}(\Omega)$ be such that $\eta=1$ in $K, \operatorname{supp}(\eta) \subset K_{\delta}$ and $0 \leq \eta \leq 1$ in $K_{\delta} \backslash K$.

Therefore, for $\psi_{\varepsilon}=\eta\left(v_{\varepsilon}-v\right)$, we have

$$
\int_{\Omega}\left|\nabla v_{\varepsilon}\right|^{p-2} \nabla v_{\varepsilon} \nabla \psi_{\varepsilon}+\left|v_{\varepsilon}\right|^{p-2} v_{\varepsilon} \psi_{\varepsilon} d x=0
$$

that is

$$
\int_{\Omega}\left|\nabla v_{\varepsilon}\right|^{p-2} \nabla v_{\varepsilon}\left(v_{\varepsilon}-v\right) \nabla \eta+\left|\nabla v_{\varepsilon}\right|^{p-2} \nabla v_{\varepsilon} \nabla\left(v_{\varepsilon}-v\right) \eta+\left|v_{\varepsilon}\right|^{p-2} v_{\varepsilon} \eta\left(v_{\varepsilon}-v\right) d x=0 .
$$

Since $v_{\varepsilon} \rightarrow v$ weakly in $W^{1, p}(\Omega)$ we have that $\left\|\nabla v_{\varepsilon}\right\|_{L^{p}(\Omega)} \leq C$, so by Hölder's inequality,

$$
\left.\left|\int_{\Omega}\right| \nabla v_{\varepsilon}\right|^{p-2} \nabla v_{\varepsilon}\left(v_{\varepsilon}-v\right) \nabla \eta d x \mid \leq\|\nabla \eta\|_{\infty} C\left\|v_{\varepsilon}-v\right\|_{L^{p}(\Omega)} .
$$

On the other hand, since $\left\|v_{\varepsilon}\right\|_{L^{p}(\Omega)} \leq C$, again by Hölder's inequality,

$$
\left.\left|\int_{\Omega}\right| v_{\varepsilon}\right|^{p-2} v_{\varepsilon} \eta\left(v_{\varepsilon}-v\right) d x \mid \leq\|\eta\|_{\infty} C\left\|v_{\varepsilon}-v\right\|_{L^{p}(\Omega)}
$$

From (4.17), (4.18) and (4.19) we obtain

$$
\lim _{\varepsilon \rightarrow 0} \int_{K_{\delta}}\left|\nabla v_{\varepsilon}\right|^{p-2} \nabla v_{\varepsilon} \nabla\left(v_{\varepsilon}-v\right) \eta d x=0 .
$$

Moreover, since $v_{\varepsilon} \rightarrow v$ weakly in $W^{1, p}(\Omega)$ we get

$$
\lim _{\varepsilon \rightarrow 0} \int_{K_{\delta}}|\nabla v|^{p-2} \nabla v \nabla\left(v_{\varepsilon}-v\right) \eta d x=0 .
$$


Combining (4.20) and (4.21) we arrive at

$$
\lim _{\varepsilon \rightarrow 0} \int_{K_{\delta}}\left(\left|\nabla v_{\varepsilon}\right|^{p-2} \nabla v_{\varepsilon}-|\nabla v|^{p-2} \nabla v\right) \nabla\left(v_{\varepsilon}-v\right) \eta d x=0 .
$$

But now, it is a well known fact (see e.g. [12]) that the integrand is nonnegative and therefore $\left(\left|\nabla v_{\varepsilon}\right|^{p-2} \nabla v_{\varepsilon}-|\nabla v|^{p-2} \nabla v\right) \nabla\left(v_{\varepsilon}-v\right) \rightarrow 0$ a.e. in $K$. From this, we can easily conclude that $\nabla v_{\varepsilon} \rightarrow \nabla v$ a.e. in $K$. Since $K$ is arbitrary in $\Omega$ we conclude the pointwise convergence of the gradients a.e. in $\Omega$.

From the pointwise convergence of the gradient the conclusion of the liminf inequality follows easily. In fact, since $J T_{\varepsilon} \rightarrow 1$ and $D T_{\varepsilon} \rightarrow I$ a.e. in $\Omega$ we have

$$
\left|\nabla v_{\varepsilon}\left(D T_{\varepsilon} \circ T_{\varepsilon}^{-1}\right)\right|^{p} J T_{\varepsilon}^{-1} \rightarrow|\nabla v|^{p} \text { a.e. in } \Omega .
$$

This last fact, together with Fatou's Lemma imply (4.16).

lim sup inequality: Given $v \in X_{\alpha}^{*}$, let $v_{k} \in \tilde{X}_{\alpha}^{\varepsilon_{k}}$ be such that $v_{k} \rightarrow v$ strongly in $W^{1, p}(\Omega)$. Observe that such a sequence exists by Lemma 4.7.

Now this and our hypotheses on $T_{\varepsilon}$ easily imply that

$$
\lim _{k \rightarrow \infty} \tilde{Q}_{\varepsilon_{k}}\left(v_{k}\right)=Q(v)
$$

The proof is completed.

Now, the proof of Theorem 4.1 follows as a simple corollary.

Proof of Theorem 4.1. The proof is now a trivial consequence of Proposition 4.9 and Theorem 4.3.

\section{Convergence of optimal Windows}

In this section we analyze the behavior of a sequence of optimal windows $\left\{\Gamma_{\varepsilon}\right\}_{\varepsilon>0}$. Recall that an optimal window is a set $\Gamma_{\varepsilon} \subset \Omega_{\varepsilon}$ such that $\left|\Gamma_{\varepsilon}\right|_{n-1}=\alpha\left|\partial \Omega_{\varepsilon}\right|_{n-1}$ and $\lambda_{\varepsilon}\left(\Gamma_{\varepsilon}\right)=\lambda_{\varepsilon}(\alpha)$ for $0<\alpha<1$.

We will see that, as a consequence of the convergence of the constants $\lambda_{\varepsilon}(\alpha) \rightarrow$ $\lambda^{*}(\alpha)$ we will deduce the convergence of these optimal windows to an optimal window of the limit problem in a suitable sense.

Theorem 5.1. Under the same assumptions and notations of Theorem 4.1, if $\Gamma_{\varepsilon} \subset \Omega_{\varepsilon}$ is an optimal window associated to $\lambda_{\varepsilon}(\alpha)$ then, up to a subsequence, it converges, as $\varepsilon \downarrow 0$, to an optimal window of the limit problem $\lambda^{*}(\alpha)$ in the following sense: Let us define the Radon measures $\left\{\nu_{\varepsilon}\right\}_{\varepsilon>0}$ as

$$
d \nu_{\varepsilon}=\chi_{\Gamma_{\varepsilon}} d S \text {. }
$$

Then, the family is pre compact in the weak topology of measures and every accumulation point of $\left\{\nu_{\varepsilon}\right\}_{\varepsilon>0}$ is of the form

$$
d \nu^{*}=\chi_{\Gamma^{*}} m d S
$$

where $\Gamma^{*}$ is an optimal windows for the problem (4.2).

In order to show the convergence of optimal windows we need a couple of lemmas. 
Lemma 5.2. Let $(X, \Sigma, \nu)$ be a measure space of finite measure and let $\left\{f_{n}\right\}_{n \in \mathbb{N}}, f$ be $\nu$-measurable nonnegative functions such that $f_{n} \rightarrow f \nu$-a.e.

Let $\left\{\mu_{n}\right\}_{n \in \mathbb{N}}$ and $\mu$ be nonnegative measures, absolutely continuous with respect to $\nu$ such that $\mu_{n}(A) \rightarrow \mu(A)$, for every $A \in \Sigma$.

Then, if $\lim _{n \rightarrow \infty} \mu_{n}\left(\left\{f_{n}=0\right\}\right)=\mu(\{f=0\})$, given $\varepsilon>0$ there exists $j_{0} \in \mathbb{N}$ such that, for every $j \geq j_{0}$,

$$
\limsup _{n \rightarrow \infty} \mu_{n}\left(\left\{0<f_{n} \leq \frac{1}{j}\right\}\right)<\varepsilon
$$

Proof. Let $\varepsilon>0$. Since $\chi_{\left\{0<f \leq \frac{1}{j}\right\}} \rightarrow 0 \nu$-a.e. when $j \rightarrow \infty$, we have that there exists $j_{0} \in \mathbb{N}$ such that

$$
\mu\left(\left\{0<f \leq \frac{1}{j}\right\}\right)<\varepsilon, \quad \text { for every } j \geq j_{0} .
$$

Since $f_{n} \rightarrow f \nu$-a.e., we have that

$$
\left\{f \leq \frac{1}{j}\right\} \supset \bigcap_{n_{0} \in \mathbb{N}} \bigcup_{k \geq n_{0}}\left\{f_{k} \leq \frac{1}{j}\right\}
$$

from where

$$
\mu\left(\left\{f \leq \frac{1}{j}\right\}\right) \geq \lim _{n_{0} \rightarrow \infty} \mu\left(\bigcup_{k \geq n_{0}}\left\{f_{k} \leq \frac{1}{j}\right\}\right) .
$$

Hence, given $\delta>0$, there exists $n_{0} \in \mathbb{N}$ such that

$$
\mu\left(\left\{f \leq \frac{1}{j}\right\}\right)+\delta \geq \mu\left(\bigcup_{k \geq n_{0}}\left\{f_{k} \leq \frac{1}{j}\right\}\right) .
$$

By our hypothesis on the convergence of the measures,

$$
\lim _{n \rightarrow \infty} \mu_{n}\left(\bigcup_{k \geq n_{0}}\left\{f_{k} \leq \frac{1}{j}\right\}\right)=\mu\left(\bigcup_{k \geq n_{0}}\left\{f_{k} \leq \frac{1}{j}\right\}\right),
$$

from where

$$
\mu\left(\bigcup_{k \geq n_{0}}\left\{f_{k} \leq \frac{1}{j}\right\}\right)+\delta \geq \mu_{n}\left(\bigcup_{k \geq n_{0}}\left\{f_{k} \leq \frac{1}{j}\right\}\right) \geq \mu_{n}\left(\left\{f_{n} \leq \frac{1}{j}\right\}\right),
$$

for any $n$ large enough.

Using (5.2) and (5.3) we obtain

$$
\mu\left(\left\{f \leq \frac{1}{j}\right\}\right)+2 \delta \geq \limsup _{n \rightarrow \infty} \mu_{n}\left(\left\{f_{n} \leq \frac{1}{j}\right\}\right)
$$

and since $\delta>0$ is arbitrary, it follows that

$$
\limsup _{n \rightarrow \infty} \mu_{n}\left(\left\{f_{n} \leq \frac{1}{j}\right\}\right) \leq \mu\left(\left\{f \leq \frac{1}{j}\right\}\right) .
$$

Now, the lemma follows from (5.1) and (5.4) by using the hypothesis

$$
\lim _{n \rightarrow \infty} \mu_{n}\left(\left\{f_{n}=0\right\}\right)=\mu(\{f=0\}) .
$$

The proof is completed. 
Lemma 5.3. Let $(X, \Sigma, \nu)$ be a measure space of finite measure and let $\left\{f_{n}\right\}_{n \in \mathbb{N}}, f$ be $\nu$-measurable nonnegative functions such that $f_{n} \rightarrow f \nu$-a.e.

Let $\left\{\mu_{n}\right\}_{n \in \mathbb{N}}$ and $\mu$ be nonnegative measures, absolutely continuous with respect to $\nu$ such that $\mu_{n}(A) \rightarrow \mu(A)$, for every $A \in \Sigma$.

Then, if $\lim _{n \rightarrow \infty} \mu_{n}\left(\left\{f_{n}=0\right\}\right)=\mu(\{f=0\})$, it follows that

$$
\lim _{n \rightarrow \infty} \mu_{n}\left(\left\{f_{n}=0\right\} \Delta\{f=0\}\right)=0 .
$$

Proof. By Egoroff's Theorem, we have that, given $\delta>0$, there exists a measurable set $C_{\delta} \subset X$ such that

$$
f_{n} \rightrightarrows f \text {, uniformly when } n \rightarrow \infty \text { in } X \backslash C_{\delta}
$$

with

$$
\mu\left(C_{\delta}\right)<\delta .
$$

Observe that, as $\mu_{n}(A) \rightarrow \mu(A)$ for every $A$ measurable, we can assume that

$$
\mu_{n}\left(C_{\delta}\right)<\delta,
$$

for every $n$ large enough.

Define now the set $E_{\delta}=X \backslash C_{\delta}$ and using this uniform convergence on the set $E_{\delta}$, we have

$$
\left\{f_{n}=0\right\} \cap E_{\delta} \subset\{f \leq \delta\} \cap E_{\delta},
$$

for $\varepsilon$ small enough.

We then have that

$$
\{f=0\} \backslash\left\{f_{n}=0\right\} \subset\left(\left(\{f \leq \delta\} \backslash\left\{f_{n}=0\right\}\right) \cap E_{\delta}\right) \cup C_{\delta},
$$

from where

$$
\mu_{n}\left(\{f=0\} \backslash\left\{f_{n}=0\right\}\right) \leq \mu_{n}(\{f \leq \delta\})-\mu_{n}\left(\left\{f_{n}=0\right\}\right)+\delta .
$$

Taking the limit as $n \rightarrow \infty$, we obtain

$$
\limsup _{n \rightarrow \infty} \mu_{n}\left(\{f=0\} \backslash\left\{f_{n}=0\right\}\right) \leq \mu(\{f \leq \delta\})-\mu(\{f=0\})+\delta,
$$

and now making $\delta \rightarrow 0$ we can conclude

$$
\lim _{n \rightarrow \infty} \mu_{n}\left(\{f=0\} \backslash\left\{f_{n}=0\right\}\right)=0 .
$$

On the other hand, given $j \in \mathbb{N}$, there exists $n_{j} \in \mathbb{N}$ such that

$$
\{f=0\} \cap E_{j} \subset\left\{f_{n_{j}}<\frac{1}{j}\right\} \cap E_{j},
$$

where $\nu\left(X \backslash E_{j}\right) \leq \frac{1}{j}$.

Now, reasoning as in the previous case,

$$
\limsup _{j \rightarrow \infty} \mu_{n_{j}}\left(\left\{f_{n_{j}}=0\right\} \backslash\{f=0\}\right) \leq \limsup _{j \rightarrow \infty}\left(\mu_{n_{j}}\left(\left\{f_{n_{j}}<\frac{1}{j}\right\}\right)\right)-\mu(\{f=0\}) .
$$

But, from Lemma 5.2, it follows that

$$
\mu_{n_{j}}\left(\left\{f_{n_{j}}<\frac{1}{j}\right\}\right)=\mu_{n_{j}}\left(\left\{f_{n_{j}}=0\right\}\right)+\mu_{n_{j}}\left(\left\{0<f_{n_{j}}<\frac{1}{j}\right\}\right) \rightarrow \mu(\{f=0\}) .
$$

This completes the proof. 
Remark 5.4. When the sequence of measures $\mu_{n}$ is constant, this Lemma was proved in [7, Lemma 3.1].

With the help of Lemma 5.3 we can now prove Theorem 5.1

Proof of Theorem 5.1. Let $u_{\varepsilon} \in W^{1, p}\left(\Omega_{\varepsilon}\right)$ be an extremal for $\lambda_{\varepsilon}(\alpha)$. We can assume that $u_{\varepsilon} \in X_{\alpha}^{\varepsilon}$. Then, by [5, Theorem 3.6], we have that $\left\{u_{\varepsilon}=0\right\} \cap \Omega_{\varepsilon}=\Gamma_{\varepsilon}$ is an optimal window for $\lambda_{\varepsilon}(\alpha)$ and hence it verifies $\left|\Gamma_{\varepsilon}\right|_{n-1}=\alpha\left|\Omega_{\varepsilon}\right|_{n-1}$.

Consider now the rescaled functions $v_{\varepsilon}:=u_{\varepsilon} \circ T_{\varepsilon}^{-1}$. Then $v_{\varepsilon}$ is an extremal of $\tilde{Q}_{\varepsilon}$ in the set $\tilde{X}_{\alpha}^{\varepsilon}$.

By Theorem 4.1, we can assume that there exists $v \in W^{1, p}(\Omega)$ such that $v_{\varepsilon} \rightarrow v$ weakly in $W^{1, p}(\Omega), v \in X_{\alpha}^{*}$ and $v$ is an extremal for $\lambda^{*}(\alpha)$. In particular

$$
\mu^{*}(\{v=0\} \cap \partial \Omega)=\alpha \mu^{*}(\partial \Omega) .
$$

On the other hand,

$$
\left|\left\{u_{\varepsilon}=0\right\} \cap \partial \Omega_{\varepsilon}\right|_{n-1}=\int_{\partial \Omega_{\varepsilon}} \chi_{\left\{u_{\varepsilon}=0\right\}} d S=\int_{\partial \Omega} \chi_{\left\{v_{\varepsilon}=0\right\}} J_{\tau} T_{\varepsilon}^{-1} d S .
$$

So, if we denote by $\mu_{\varepsilon}$ to the measure $d \mu_{\varepsilon}=J_{\tau} T_{\varepsilon}^{-1} d S$ on $\partial \Omega$, we have that

$$
\mu_{\varepsilon}\left(\left\{v_{\varepsilon}=0\right\} \cap \partial \Omega\right)=\alpha\left|\partial \Omega_{\varepsilon}\right|_{n-1}=\alpha \mu_{\varepsilon}(\partial \Omega),
$$

and since $J_{\tau} T_{\varepsilon}^{-1} \stackrel{*}{\rightarrow} m$ weakly-* in $L^{\infty}(\partial \Omega)$, it holds that

$$
\mu_{\varepsilon}(A) \rightarrow \mu^{*}(A)
$$

for every $A \subset \partial \Omega$ measurable. In particular, $\mu_{\varepsilon}(\partial \Omega) \rightarrow \mu^{*}(\partial \Omega)$.

All of this discussion leads us to conclude that

$$
\mu_{\varepsilon}\left(\left\{v_{\varepsilon}=0\right\} \cap \partial \Omega\right) \rightarrow \mu^{*}(\{v=0\} \cap \partial \Omega) .
$$

Now we are in a position of applying Lemma 5.3 and conclude that

$$
\mu_{\varepsilon}\left(\left[\left\{v_{\varepsilon}=0\right\} \triangle\{v=0\}\right] \cap \partial \Omega\right) \rightarrow 0 .
$$

Now, let $\Gamma_{\varepsilon}$ be an optimal window and let $u_{\varepsilon} \in X_{\alpha}^{\varepsilon}$ an associated extremal. Let $v_{\varepsilon}=u_{\varepsilon} \circ T_{\varepsilon}^{-1}$ the rescaled extremal as was previously described. Again, we can assume that $v_{\varepsilon} \rightarrow v$ a.e. in $\partial \Omega$ where $v \in X_{\alpha}^{*}$ is an extremal associated to $\lambda^{*}(\alpha)$.

Let $f \in C_{b}\left(\mathbb{R}^{n}\right)$, then

$$
\begin{aligned}
\int f d \nu_{\varepsilon}-\int f d \nu^{*}= & \int_{\partial \Omega_{\varepsilon}} f \chi_{\left\{u_{\varepsilon}=0\right\}} d S-\int_{\partial \Omega} f \chi_{\{v=0\}} m d S \\
= & \int_{\partial \Omega}\left(f \circ T_{\varepsilon}^{-1}\right) \chi_{\left\{v_{\varepsilon}=0\right\}} d \mu_{\varepsilon}-\int_{\partial \Omega} f \chi_{\{v=0\}} d \mu^{*} \\
= & \int_{\partial \Omega}\left(\chi_{\left\{v_{\varepsilon}=0\right\}}-\chi_{\{v=0\}}\right)\left(f \circ T_{\varepsilon}^{-1}\right) d \mu_{\varepsilon} \\
& +\int_{\partial \Omega} \chi_{\{v=0\}}\left[\left(f \circ T_{\varepsilon}^{-1}\right)-f\right] d \mu_{\varepsilon} \\
& +\int_{\partial \Omega} \chi_{\{v=0\}} f\left(d \mu_{\varepsilon}-d \mu^{*}\right) \\
= & A_{\varepsilon}+B_{\varepsilon}+C_{\varepsilon} .
\end{aligned}
$$


Each of these terms can be easily shown to converge to zero. In fact

$$
\left|A_{\varepsilon}\right| \leq\|f\|_{\infty} \mu_{\varepsilon}\left(\left[\left\{v_{\varepsilon}=0\right\} \triangle\{v=0\}\right] \cap \partial \Omega\right) \rightarrow 0,
$$

by Lemma 5.3. On the other hand,

$$
\left|B_{\varepsilon}\right| \leq\left\|\left(f \circ T_{\varepsilon}^{-1}\right)-f\right\|_{L^{\infty}(\partial \Omega)} \mu_{\varepsilon}(\partial \Omega) \rightarrow 0,
$$

since $\mu_{\varepsilon}(\partial \Omega)$ is convergent (hence bounded) and $f \circ T_{\varepsilon}^{-1} \rightrightarrows f$ on compact sets.

Finally, using that $\mu_{\varepsilon} \rightarrow \mu^{*}$ weakly in the sense of measures it follow that

$$
\left|C_{\varepsilon}\right| \rightarrow 0 \text {. }
$$

This completes the proof of the theorem.

\section{ACKNOWLEDGEMENTS}

This paper was partially supported by Universidad de Buenos Aires under grant UBACyT 20020130100283BA, by CONICET under grant PIP 2009 845/10 and by ANPCyT under grant PICT 2012-0153. J. Fernández Bonder is a member of CONICET and J.F. Spedaletti is a doctoral fellow of CONICET.

We thank the referees for the throughout reading of the manuscript and for some suggestions that help to improve the presentation of the paper and we thank Leandro Del Pezzo for his help with the figure.

\section{REFERENCES}

1. A. Bensoussan, J.-L. Lions, and G. Papanicolaou, Asymptotic analysis for periodic structures, AMS Chelsea Publishing, Providence, RI, 2011, Corrected reprint of the 1978 original [MR0503330]. MR 2839402

2. Andrea Braides, $\Gamma$-convergence for beginners, Oxford Lecture Series in Mathematics and its Applications, vol. 22, Oxford University Press, Oxford, 2002. MR 1968440 (2004e:49001)

3. Doina Cioranescu and François Murat, A strange term coming from nowhere, Topics in the mathematical modelling of composite materials, Progr. Nonlinear Differential Equations Appl., vol. 31, Birkhäuser Boston, Boston, MA, 1997, pp. 45-93. MR 1493040

4. Gianni Dal Maso, An introduction to $\Gamma$-convergence, Progress in Nonlinear Differential Equations and their Applications, 8, Birkhäuser Boston, Inc., Boston, MA, 1993. MR 1201152 (94a:49001)

5. Leandro Del Pezzo, Julián Fernández Bonder, and Wladimir Neves, Optimal boundary holes for the Sobolev trace constant, J. Differential Equations 251 (2011), no. 8, 2327-2351. MR 2823670 (2012g:49102)

6. Jochen Denzler, Windows of given area with minimal heat diffusion, Trans. Amer. Math. Soc. 351 (1999), no. 2, 569-580. MR 1475680 (99d:49076)

7. Julián Fernández Bonder, Pablo Groisman, and Julio D. Rossi, Optimization of the first Steklov eigenvalue in domains with holes: a shape derivative approach, Ann. Mat. Pura Appl. (4) 186 (2007), no. 2, 341-358. MR 2295124 (2007m:35179)

8. Julián Fernández Bonder, Rafael Orive, and Julio D. Rossi, The best Sobolev trace constant in a domain with oscillating boundary, Nonlinear Anal. 67 (2007), no. 4, 1173-1180. MR 2325371 (2008g:35010)

9. Julián Fernández Bonder and Julio D. Rossi, Existence results for the p-Laplacian with nonlinear boundary conditions, J. Math. Anal. Appl. 263 (2001), no. 1, 195-223. MR 1864315 (2002h:35063)

10. Antoine Henrot and Michel Pierre, Variation et optimisation de formes, Mathématiques \& Applications (Berlin) [Mathematics \& Applications], vol. 48, Springer, Berlin, 2005, Une analyse géométrique. [A geometric analysis]. MR 2512810 (2009m:49003)

11. Enrique Sánchez-Palencia, Nonhomogeneous media and vibration theory, Lecture Notes in Physics, vol. 127, Springer-Verlag, Berlin-New York, 1980. MR 578345 (82j:35010) 
12. Jacques Simon, Régularité de la solution d'une équation non linéaire dans $\mathbf{R}^{N}$, Journées d'Analyse Non Linéaire (Proc. Conf., Besançon, 1977), Lecture Notes in Math., vol. 665, Springer, Berlin, 1978, pp. 205-227. MR 519432 (80b:35035)

(J. Fernández Bonder) Departamento de Matemática fCEN - Universidad de Buenos Aires and imas - COniCet. Ciudad Universitaria, Pabellón I (C1428EGA) Av. Cantilo 2160. Buenos Aires, Argentina.

(J. F. Spedaletti) Departamento de Matemática, Universidad Nacional de San Luis and imASL - CONiCET. EjÉrcito de los Andes 950 (D5700HHW), San Luis, Argentina.

E-mail address, J. Fernandez Bonder: jfbonder@dm.uba.ar

$U R L$, J. Fernandez Bonder: http://mate.dm.uba.ar/〜jfbonder

E-mail address, J. F. Spedaletti: jfspedaletti@unsl.edu.ar 